\title{
Horus, el huérfano
}

\author{
Helena DÍAZ RIVAS
}

Horus es una de las divinidades más importantes del panteón egipcio, personificación del heredero legítimo al trono y del que todo faraón era la encarnación terrestre. Pero solemos pasar por alto los aspectos humanos de esta divinidad, como por ejemplo su condición de huérfano. Horus, el hijo de Isis, es amamantado, criado y protegido por ésta. Horus, el hijo de Osiris, es el heredero de su padre. Pero uno de los personajes que acompañan a la figura de Horus y que condiciona su existencia es Seth, su contendiente. Tradicionalmente se ha atribuido la debilidad de Horus a su condición de infante por carecer de las cualidades propias de un adulto para vencer a este enemigo. Sin negar estas carencias de la niñez, la ausencia de referente paterno puede ser otra de las causas de la debilidad de Horus frente a Seth. En una sociedad como la egipcia, la madre era la encargada de criar y de proteger al niño; y el padre el responsable de educar y de transmitir la sabiduría y los preceptos morales al hijo. Horus tuvo protectora, pero no tuvo educador. Nuestra intención es indagar en la orfandad de Horus, un condicionante importante en la trayectoria de esta divinidad.

\section{Horus, the Orphan}

Horus is probably one of the most famous divinities in the Egyptian pantheon, the legitimate heir to the throne and the incarnation of pharaoh. Even so, the human aspects of this divinity, for example the fact of his being an orphan, are ignored by most of us. Horus, as son of Isis, is nursed and protected by his mother. Horus, as son of Osiris, is the heir of his father. But one of the figures who accompanies Horus, and who conditions his existence, is Seth, his contender. The weakness of Horus has been attributed traditionally to his condition as an infant, therefore lacking in those adult qualities necessary to overcome his enemy. Without denying the deficiencies of his childhood, the absence of a paternal referent could be another reason for the weakness of Horus, in respect of Seth. In a society like the ancient Egyptian, the mother would have been responsible for raising and protecting the children; and it would be the father's responsibility to educate, transmitting wisdom and moral precepts to the son. Horus had a protector, but did not have educator. Our intention is to examine the orphan state of Horus, a conditioning factor in the development of this divinity.

KEY WORDS: Horus, orphanship, archetype, mother

$\mathrm{U}$ no de los temas sociales menos abordado del antiguo Egipto es el de la orfandad. El desarrollo de este tipo de estudios en otros ámbitos culturales es bastante amplio por lo que llama la atención la ausencia a este respecto en nuestra disciplina y más si se tiene presente que una de las divinidades centrales de la cultura egipcia, Horus, era huérfano. Desde el punto de vista de la historia de las religiones, también existe gran variedad de estudios sobre el arquetipo de la divinidad infantil, que a menudo suele ser huérfana $y$, sin embargo, el vacío es nuevamente la respuesta egiptológica.

El niño tiene un papel destacado en la mitología, puesto que es un símbolo universal. Por un lado, su nacimiento evoca la creación y es una imagen adecuada para la regeneración; y

Fecha de recepción: 25 de enero de 2007

Fecha de admisión: 15 de junio de 2007 
por otro, es el elemento que pone en contacto el pasado y el futuro. Kerényi establece una relación directa entre el momento de la creación del cosmos y el niño primordial ${ }^{1}$ en su absoluta soledad cósmica ${ }^{2}$, una característica propia del elemento original.

Además del niño primordial, se encuentran gran variedad de divinidades infantiles. No todas ellas poseen un mito de nacimiento e infancia, sin embargo muchas de las que cuentan con uno responden al arquetipo del niño divino. Este modelo mítico presenta unos rasgos distintivos fundamentales. $\mathrm{Su}$ concepción o nacimiento suele ser milagro$\mathrm{so}^{3}$, acostumbra a ser huérfano, bien de padre o de ambos progenitores, bien es un niño abandonado ${ }^{4}$, que al fin y al cabo es otra forma de orfandad. Diversos peligros le acechan durante su infancia ${ }^{5}$; no obstante, su destino está predestinado ${ }^{6}$ y lo alcanza a través de la venganza ${ }^{7}$. La mayoría de estas divinidades presentan además poderes extraordinarios, fuerza y sabiduría ${ }^{8}$. Esta descripción también remite indudablemente a la mitología del héroe ${ }^{9}$, cuyos comienzos dramáticos se compensan con la "grandeza" de su futuro; ésta es la suerte que sufrieron personajes como Rómulo y Remo o Moisés.

Como les sucede a otras divinidades, la concepción y el nacimiento de Horus no pueden sino calificarse de milagrosos ${ }^{10}$. Osiris es una divinidad muerta a la que Isis "revive" para concebir. En un himno dedicado a Osiris, la estela del Louvre C286 se dice, "(es ella) quien levantó al que estaba inerte, al de corazón cansado, (y es ella) quien recibió su semen (y) engendró un heredero"11. Por lo tanto, Horus es huérfano incluso antes de nacer, circunstancia que queda reflejada en el Libro de los Muertos cuando él mismo alude a su condición, "Oh, Nut, yo soy el heredero del cielo [...] Yo salí del vientre siendo huérfano de

1. Jung y Kerényi, 2004: 43-45, 67-68. Ver también Clift, en Eliade, 1987: 243-245. En la cosmogonía hermopolitana, por ejemplo, sobre la Colina primigenia surgió una flor de loto o de un huevo, del que emergió un Niño primordial que se convirtió en el Sol.

2. Eliade, 1981: 259.

3. Un dios de los vogules (Hungría) denominado "el hombre que contempla el mundo" nació del flanco derecho de su madre; Michabo, el nieto de la Luna, según la mitología de los algonquines (Norteamérica), era hijo del Viento de Oeste y una virgen y fue concebido gracias a la brisa. Jesús nace de la Virgen María y del Espíritu Santo.

4. El niño Pan, de la mitología griega, es abandonado por su madre y su nodriza. Zeus, según la tradición cretense del mito, es dejado en una cueva del monte Dicte donde había nacido o en el monte Liceo según se recoge en la versión arcadia.

5. El niño Jesús se ve amenazado por el edicto promulgado por Herodes. Crono devora a sus hijos ante la amenaza de ser destronado.

6. Un claro ejemplo es el de Horus, tal y como se señala en los Textos de los Sarcófagos, CT 148, II 212-213. Isis anuncia el destino de su hijo antes de que éste haya nacido.

7. Esta venganza no debe entenderse siempre de forma textual puesto que en algunas ocasiones la victoria de estos personajes vienen a encarnar cambios significativos para la humanidad, como son los casos de Jesús o de Gautama Buda.

8. Ésta es la única característica que a priori Horus no cumple, por lo tanto se dejará esta cuestión abierta y será tratada en otra ocasión.

9. Eliade, 2003: 41-55. Al respecto ver también Gaster, en Eliade, 1987: 302-305.

10. Ver nota 3.

11. Estela del Louvre C286, línea 16 sțšt $n n w n$ wrd ib hnpt $m w=$ f irt $i w^{\top} w$ en Moret, 1931:743 y pl. II. Para Reino Antiguo y Medio ver respectivamente PT 336, Pyr. 632 ${ }^{\mathrm{TPMN}}$; PT 593, Pyr. 1634 ${ }^{\mathrm{MN}}$ y CT 148, II 209-211. 
padre (y) mi sabiduría era inexistente como responsable de mis actos"12.

Según Kerényi el tema del huérfano tendría dos variantes, una en la que el niño se presenta en estado de completa soledad y otra en la que la madre participa del sentimiento de abandono y soledad $^{13}$. Al considerar el caso egipcio se aprecia que la segunda variante prevalece sobre la primera. La presencia de Isis en este contexto representa el arquetipo de la madre que amaman$\mathrm{ta}^{14}$, cría y protege a su hijo ${ }^{15}$. Al igual que otras divinidades femeninas, aunque éstas en menor medida $^{16}$, ella es la encargada del cuidado de Horus. En la estela antes mencionada (Louvre C286) se afirma que Isis era "quien amamantó al niño en soledad sin que su lugar fuera conocido"17. Y en el Libro de los Muertos se pone de manifiesto su carácter protector: "Isis ha venido, se ha detenido en la ciudad, ha buscado un escondite para Horus; cuando salió de las malezas de los pantanos del Delta, su corazón estaba ulcerado, su sien estaba sombría ${ }^{18}$ y le proporcionaron protección [...]. Su madre, la gran diosa, establece su protección y acecha a los que vengan contra Horus"19. Un documento tardío, la estela de Metternich, describe un momento especialmente dramático cuando en ausencia de Isis, Horus es picado por un escorpión ${ }^{20}$; a su regreso, ella muestra su desesperación ante su completa soledad "mi temor era grande, porque nadie acudió al oír mi voz, mi padre estaba en el mundo subterráneo, mi madre en el cementerio, mi hermano estaba en el ataúd, el otro era un enemigo que insistía en su odio contra mí y mi hermana menor estaba en su casa" 21 .

Otra cuestión interesante es el lugar en el que el niño nace, es abandonado o se esconde, puesto que acostumbran a ser parajes inhóspitos ${ }^{22}$. Desde el nacimiento y durante la infancia es acechado por peligros que se manifiestan de formas

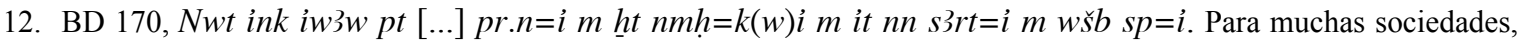
entre ellas la egipcia, los niños eran concebidos como seres incompletos. Tal como señala Lichtheim, 1997: 16, las carencias fundamentales asociadas a la infancia eran la ausencia de conocimiento y de entendimiento. Se debe prestar especial atención a la acepción utilizada en este caso wšb sp, $\mathrm{Wb}$. I, 372, 1, "ser responsable", pero en referencia directa al comportamiento que el buen hijo debe tener. La ausencia de sabiduría de Horus no es solamente la inherente a la infancia, sino que atañe al desconocimiento de las actitudes que el hijo debe adoptar con respecto a sus progenitores. Si se tiene en cuenta la función que el padre desempeñaba como encargado de la transmisión del conocimiento, de los valores morales y de las normas de conducta, se puede inferir que Horus está enfatizando su debilidad por su condición de huérfano, puesto que no tiene un modelo conductual, desconoce cuáles son sus obligaciones y sus responsabilidades en relación a sus padres.

13. Jung y Kerényi, 2004: 46-47.

14. PT 689, Pyr. 2089a ${ }^{\mathrm{N}}$; CT 334, IV $182 \mathrm{e}-\mathrm{h}$.

15. CT 148, II 225b; Ver también nota 19.

16. Neftis y Sejat-Hor, PT 555, Pyr. $1375 \mathrm{a}^{\mathrm{MN}}$, Nejbet CT 16-17, I 48; Según Kerényi, la presencia de nodrizas divinas enfatiza que, pese a su orfandad, el niño es un ser amado y protegido por las divinidades. Ver Jung y Kerényi, 2004: 47.

17. Estela del Louvre C286, línea 16. $\breve{s} d t h h n(w) m w^{\top} w n r h(w) b w=f i m$ en Moret, 1931: 743.

18. Según Barguet se puede tratar de la debilidad del joven Horus. Barguet, 2000: 225, nota 5.

19. BD 157, traducido por Barguet, 2000: 225.

20. Hay que destacar que el momento de máxima vulnerabilidad y peligro para Horus se produce precisamente en ausencia de su madre.

21. Assmann, 2001: 132-133. Brunner-Traut, 2000: 153-155.

22. Leto y Apolo en la isla de Delos o el ejemplo antes comentado de Zeus. Mucho más frecuentes son los casos entre los héroes mitológicos, como refleja la epopeya de Kullervo y su madre Kalervo en la patria de Untamo (hermano y asesino del padre de Kullervo) recogida en el canto XXXI del Kalevala; ver también Eliade, 1981: 259. 
diversas, fuerzas hostiles de la naturaleza o animales que simbolizan a la divinidad de la que se oculta y que ha causado su orfandad.

Según el mito egipcio, como es sabido, Isis da a luz a Horus en las marismas pantanosas de Quemmis $^{23}$, una localidad situada en el Delta, donde ambos se esconden ${ }^{24}$. Horus, desde su nacimiento, está en un entorno caótico. Una declaración de los Textos de las Pirámides refleja esta circunstancia "el Nun dio a luz al rey en su mano derecha, un niño cuya sabiduría era inexistente, él (el Nun) protegió al rey de los dioses malvados, él no dará al rey a los dioses malvados" 25 . De la misma manera que Horus nace en Quemmis el monarca nace en el Nun, nace en el caos, el Nun le protege de sus enemigos, como la frondosidad de las marismas protegieron a Horus de Seth. El soberano, como su encarnación se está identificando con la divinidad huérfana e indefensa que carece de sabiduría ${ }^{26}$. Otro pasaje, esta vez procedente de los Textos de los Sarcófagos, es más concreto al evidenciar el peligro: "que no venga este enemigo que mató a su padre, de otra manera él destruirá el huevo en su infancia" ${ }^{27}$.
El mito adquiere una significación especial cuando el niño divino se revela como vengador ante la afrenta que se ha cometido contra él. La venganza desencadena una contienda. El resultado de su lucha y su victoria es el establecimiento de un nuevo régimen del mundo ${ }^{28}$.

En el antiguo Egipto coexisten dos ciclos mitológicos, en origen diferenciados, que nos remiten al conflicto ${ }^{29}$. Uno de ellos es la contienda entre Horus y Seth, en la que ambos pugnan por la sucesión al trono, expresada en términos jurídicos y en la que la "resolución judicial" constituye la venganza. El otro, es la lucha de los antagonistas cósmicos, tan característica en las culturas de discurso mítico ${ }^{30}$. Frente al enunciado judicial, basado en el binomio herencia-legitimidad, en el que Horus cumpliría el papel de niño huérfano, ayudado en ocasiones por su madre o por otras divinidades, se encuentra a Horus como divinidad cósmica que lucha cuerpo a cuerpo contra Seth ${ }^{31}$. En este caso, la pugna ha de entenderse en parámetros de la dialéctica mitológica orden-caos ${ }^{32}$.

En el primer enunciado, la injusticia cometida sobre Horus es el asesinato de su padre, lo que

23. En los CT 286, IV 37 se señala que "él (Horus) fue concebido en Buto (y) fue parido en Quemmis" iwr $(w)=f m P$ $m s(w)=f m$ 3h-byt. Otras referencias al lugar de nacimiento en: PT 519, Pyr.1214b ${ }^{\mathrm{PMN}}$ PT 609, Pyr. 1703c $\mathrm{c}^{\mathrm{M}}$; PT 701, Pyr. $2190 \mathrm{a}^{\mathrm{N}}$.

24. CT 148, II 217, Estela de Metternich, en Assmann, 2001: 132-133; estela del año 6 de Taharqa, en Assmann, 2001: 98.

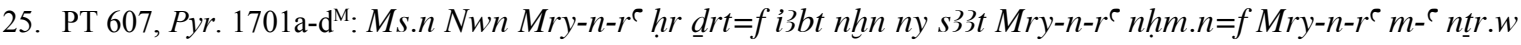

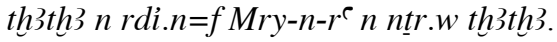

[ 26. Ver nota 12.

27. CT 148, II 217e-f, mi iw rqw pw sm3 it $=f s \underline{d}=f$ swht $m-h \underline{h} w$ $n h n(w)=s$, ver Assmann, 2001: 131. Para otra traducción ver Faulkner, 1969: 216.

28. Jung y Kerényi, 2004: 84.

29. Los dos ciclos eran originariamente independientes, pero en las fuentes textuales a menudo aparecen entremezclados, ver por ejemplo CT 7-9, I 19-33.

30. Según la visión dualista del cosmos basado en los principios antagónicos, como en la mitología mongola, ver al respecto Fedotoff, 1996: 201-223; para la mitología africana ver Cervelló, 1996: 150-151; para el caso egipcio también ver Assmann, 2001: 134-137.

31. PT 570, Pyr.1463 ${ }^{\mathrm{PM}}$. Algunas alusiones al ojo de Horus y los testículos de Seth, PT 359, Pyr. 594 ${ }^{\mathrm{TPN}}$; PT 475, Pyr. 946 ${ }^{\mathrm{PMN}}$; CT 9, I 30; BD 17.

32. Ver Frankfort, 2001: 44; Cervelló, 1996: 140. 
hace peligrar el ascenso al trono de su hijo y sucesor puesto que Seth, en su condición de hermano de Osiris, lo reclama para sí. En los Textos de los Sarcófagos se ejemplifica la causa y al causante de la injuria:

Saludos a vosotros portales, cuyos nombres son secretos, cuyos lugares son sagrados. Proteged a esta $\mathrm{N}$ ante cada malvado obstáculo de los poderosos que están en vuestra presencia, para que esta $\mathrm{N}$ llegue ante el Señor de Todo. Ella ha pacificado a los dos contendientes. Esta $\mathrm{N}$ ha hecho ascender al huérfano, (para que) pueda quejarse a causa de la injuria que ha sido hecha contra su padre por Tebeh $^{33}$, quien descuartizó sus miembros ${ }^{34}$.

En este escenario judicial ${ }^{35}$, Horus, hijo de Osiris, es débil, pero está amparado por la ley y Seth es fuerte pero carece de legitimidad. El comienzo de la Contienda entre Horus y Seth es significativo a este respecto, puesto que en él se indica la condición infantil del primero "había un niño (divino)" "36. A lo largo del relato se ve la incapacidad de éste para valerse por sí mismo y cómo su madre Isis le protege y ayuda ante $\mathrm{Seth}^{37}$.

En los Textos de las Pirámides también se constata la búsqueda de justicia cuando se dice:

Oh! Gueb, toro de Nut, este Horus es Unis, heredero de su padre. Este Unis ha ido y ha vuelto, el cuarto de estos cuatro dioses, que ha traído agua $[\ldots]$ que ha causado júbilo en la fuerza de sus antepasados. Él desea su justificación por lo que él ha hecho. Unis el huérfano buscaba justicia frente a la orfandad. Las Dos Verdades juzgaron, (él) estaba desprovisto de testigos. Las Dos Verdades ordenaron que los tronos de Gueb volvieran a él ${ }^{38}$.

Esta búsqueda podría ser entendida además como una petición de ayuda frente a una posición desfavorable. La orfandad es en este caso la causa del perjuicio que sufre Horus. En los Textos de los Sarcófagos se encuentra el mismo pasaje con una variante interesante. "Oh! Gueb, toro de Nut, prepara el camino para esta $\mathrm{N}$, (que) está sobre este camino tuyo. Esta $\mathrm{N}$ es Horus, el heredero de su padre Osiris [...]"39. El ruego de Horus no se limita en este caso a pedir ayuda por su posición desfavorable. Horus pide a Gueb que le prepare el camino. Estos pasajes no presentan a la gran divinidad urania todopoderosa, sino al vulnerable niño que necesita ser ayudado para superar sus limitaciones. Sin embargo, el hecho más relevante de este fragmento es el destinatario de los ruegos. $\mathrm{Y}$ es interesante por dos razones: por una parte, Horus recurre a otra figura masculina, Gueb, y por otra, porque en términos familiares esta divinidad es el pariente masculino más cercano al que puede recurrir, puesto que su tío es su enemigo. La falta del referente paterno hace que Horus se dirija a la generación inmediatamente anterior, acudiendo

33. D四 $T b h W b . \mathrm{V}, 262,7$, nombre de Seth.

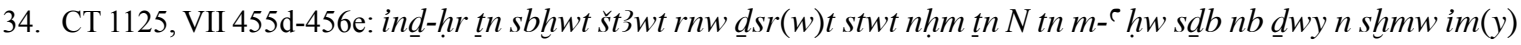

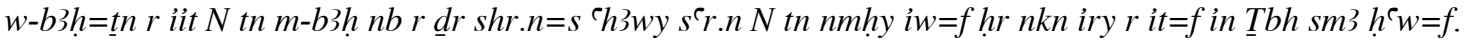
Ver también Lesko: 126.

35. Que el escenario sea esencialmente judicial no excluye la posibilidad de actos violentos entre las dos divinidades, como en el caso de La contienda entre Horus y Seth; esto es debido, por un lado, a que Seth es un dios violento y por otro, a que la debilidad de Horus sería una muestra de su falta de preparación para acceder al trono.

36. pChester Beatty I, 1,2: w`n ms, según la traducción en Campagno, 2004: 37, nota 2. Ver también López, 2005 : 164.

37. Ver Campagno, 2004: 37-63.

38. PT 260, Pyr. 316a-317cw: $i$ Gb $k$ 3 Nwt Hr pi Wnis iw ${ }^{\top}(w)$ it=f Wnis pi izy lïy fdnw $n$ fdnw ipw ntrw inw mw

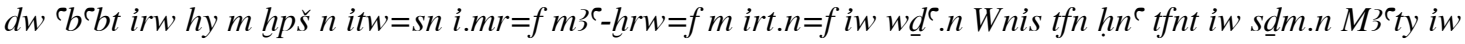
šw(=f) $m$ mtrw iw wd.n Mз`ty phr $n=f n s t w G b \underline{t} z y=f$.

39. CT 575, VI 185b-d: $i$ Gb k3 Nwt ir w3t n $N$ tn hrr(t) w3t=k tw $N$ tn hr iw $w^{\complement} w i t=f W \operatorname{sir}$. 
por lo tanto, a su abuelo. Pero Gueb es además la fuente garante de su legitimidad por vínculo sanguíneo. Como puede observarse en otra declaración de los Textos de las Pirámides "Gueb vio tu naturaleza -la de Osiris- (y) te colocó en tu lugar/trono" 40 , fue Gueb quien dio el trono a Osiris y quien puede reconocer los derechos del heredero.

La ausencia del padre plantea una cuestión interesante que ha asomado pero que aún no ha sido analizada en profundidad, y es el tema de la educación y de la transmisión del conocimiento. Plutarco en su obra Sobre Isis y Osiris relata que:

Llegado Osiris del Hades junto a Horus, lo ejercitaba y entrenaba para la batalla; después le preguntó qué es lo que consideraba más hermoso; y al decirle él 'vengar al padre y a la madre de uno por haber sido maltratados' le preguntó en segundo lugar cuál es el animal que consideraba más útil para quienes van al combate; y al responderle Horus 'el caballo', se quedó sorprendido y preguntó por qué no más el león que el caballo; pues bien, Horus le respondió que el león era útil para quien necesitaba defensa, pero el caballo para dispersar y aniquilar al enemigo en retirada. Osiris se alegró de oírlo, porque consideraba que Horus estaba lo suficientemente preparado ${ }^{41}$.

Este enunciado responde al prototipo de padre que enseña a su hijo. Horus recibe una preparación física, lo que le convierte en "grande de fuerza" y demuestra su madurez mental al responder razonablemente a las preguntas del padre, lo que se supone le hace apto para reinar. En los textos sagrados de época egipcia no se encuentra una aseveración parecida; Horus no recibe ninguna enseñanza de Osiris. Hay que re- mitirse a los textos profanos para fundamentar la transmisión del conocimiento en el ámbito de la realeza. Dos documentos fundamentales recogen este argumento, las Enseñanzas para Merikare y las Enseñanzas de Amenemhat. En ellas, el monarca difunto, Osiris, lega a su hijo los conocimientos necesarios "para que pueda ser el rey de la tierra" ${ }^{42}$. La enseñanza principal que el segundo debe aprender queda recogida en un fragmento de las Enseñanzas para Merikare "emula a tus padres, tus antepasados"43. La profundidad de este pensamiento para la civilización egipcia y por extensión para las sociedades de discurso mítico, tal y como señala Eliade, radica en que:

no es lo que ha hecho el padre y el abuelo lo que se enseña al niño, sino lo que hicieron por primera vez los antepasados míticos. Es cierto que el padre y el abuelo no han hecho otra cosa que imitar a los antepasados ${ }^{44}$.

Lo que los ancestros (las divinidades) hicieron fue establecer el cosmos tal y como los egipcios lo concebían, lo que Horus tenía que hacer era restablecer el equilibrio cósmico que había sido perturbado por Seth cuando asesinó a Osiris.

\section{Conclusiones}

El patrón social y el modelo familiar del mundo profano, pueden fácilmente reconocerse en la esfera sagrada puesto que están proyectados mitológicamente. Las figuras que sufren y están desamparadas, las viudas y los huérfanos, están representadas por Isis, la viuda que se lamenta por su esposo Osiris, y Horus el vástago huérfano.

40. PT 356, Pyr. 576c ${ }^{\mathrm{TPMN}}: m 3 . n G b q d=k d i=f t w m s t=k$; PT 371, Pyr. 649d $\mathrm{d}^{\mathrm{TPM}} m 33 . n$ Gb $q d=k d i . n=f t w m s t=k$.

41. Plut, Isis y Osiris, 19. Trad.: Pordomingo y Fernández, Madrid, 1995: 94.

42. Parkison, 1997: 206.

43. Parkison, 1997: 218.

44. Eliade, 2000: 39 . 
La niñez de Horus es la única plasmada en la mitología egipcia; aunque existen otras divinidades que se presentan en formas infantiles ${ }^{45}$, ninguna de ellas cuenta con episodios pormenorizados de sus vivencias en los primeros años de vida. Y esto es debido al papel de Horus dentro de las creencias religiosas ${ }^{46}$. Su nacimiento evoca la recreación del cosmos y su existencia es la garantía del restablecimiento y de la continuidad del orden que había sido perturbado por Seth. Su destino es gobernar Egipto; la "historia de su vida" es la historia del cosmos, la lucha y la "victoria" del orden frente al caos.

El rasgo fundamental que define la infancia de Horus es la ausencia de su padre. Pese a contar con una madre protectora, Horus es denominado huérfano. Tal como reflejan los textos sagrados y como ratifican los documentos privados ${ }^{47}$, huérfano es aquél que no tiene padre ${ }^{48}$. La base en que se sustenta este pensamiento radica en la posición que ocupan el hombre y la mujer en la sociedad. La diferencia entre la protección femenina y la masculina es la contraposición entre "lo natural" y "lo legal". La protección de la madre es natural y visceral, la que ejerce la mujer haciendo valer todas las armas de que dispone, mientras que la masculina, es la protección racional y legal, la que el hombre ejerce desde su posición de autoridad y de dominio de la sociedad. Por lo tanto la au- sencia que es significativa desde el punto de vista social, es la paterna.

La herencia recibida por Horus, el gobierno de Egipto, se debe a su legitimidad y ésta le viene dada por línea paterna. En este contexto llama la atención que en las continuas alusiones a la filiación de las divinidades, "Osiris hijo de Gueb"49, "Horus hijo de Osiris" 50 , Seth no presenta esta filiación, sus referencias filiales de siempre son en relación a Nut, su madre, puesto que carece de legitimidad.

El papel de Osiris, es total y absolutamente pasivo, como rey-muerto es el encargado de garantizar la abundancia y el bienestar de los vivos, pero no interviene en las desventuras de su hijo, puesto que como progenitor sólo legitima a su descendiente. Los casos en los que Osiris aparece, como en La contienda entre Horus y Seth ${ }^{51}$, no lo hace en "calidad de padre" sino "de juez", ajustándose a los parámetros de legales y no familiares. El papel activo lo juegan Isis, primero, como viuda que se lamenta, revive y concibe al heredero al cual protege; Gueb, como figura masculina que salvaguarda legalmente a Horus y el propio Horus, que en su condición de hijo tiene ciertos deberes que ha de cumplir, debe ascender al trono, ocupando el lugar que le corresponde por su herencia y encargarse del culto funerario de su progenitor.

45. Como Jonsu, Ihy o Shed.

46. Tal y como señala Hornung, 1999: 134, Horus se ve sometido a un proceso de envejecimiento. Si bien es cierto que otras divinidades aparecen en forma infantil, adulta y anciana, la descripción y desarrollo de sus vidas en ningún caso se hace de manera "secuenciada".

47. En las autobiografías, en las instrucciones y en los textos literarios.

48. Un ejemplo clarificador se documenta en el Oasita elocuente, cuando éste le dice a Rensi, "puesto que tu eres el padre del huérfano, el marido de la viuda, el hermano de la divorciada y la protección (lit. la falda) del que no tiene madre",

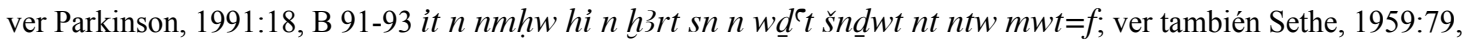
línea 13 estela de Mentu-user; Sethe, Urk IV, 972, 9 las alabanzas a Intef.

49. Osiris hijo de Gueb, ver por ejemplo PT 650, Pyr. $1833 \mathrm{c}^{\mathrm{N}}$; CT $80 \mathrm{II} 40 \mathrm{~h}$.

50. Horus hijo de Osiris, ver por ejemplo PT 437, Pyr. 975c $\mathrm{c}^{\mathrm{PMN}}$; CT 51, I 236h; CT 313, IV 91e.

51. Ver Campagno, 2004: 135-137. 


\section{Bibliografía}

Assmann, J.

2001 The Search for God in Ancient Egypt New York.

BARguet, P.

2000 El Libro de los muertos de los antiguos egipcios. Bilbao.

BRUNNER-Traut, E.

2000 Cuentos del Antiguo Egipto. Buenos Aires.

BucK, A. DE

1935-1961 The Egyptian Coffin Texts. Chicago, 7 vols.

Campagno, M.

2004 La contienda entre Horus y Set. Buenos Aires.

Cervelló, J.

1996 Egipto y África. Origen de la civilización $y$ de monarquía faraónica en su contexto Africano. (AuOr-S 13). Sabadell.

Clift, W. B.

1987 Child, en Eliade, M. (ed.): The Encyclopedia of Religions, Vol. III, New York: 243-245.

Eliade, M.

1981 Tratado de historia de las religiones. Morfología y dinámica de lo sagrado. Madrid.

2000 Aspectos del mito. Barcelona.

2003 El mito del eterno retorno, arquetipos y repetición. Madrid.

FAULKNER, R. O.

1969 The Ancient Egyptian Pyramid Texts. Oxford.

2004 The Ancient Egyptian Coffin Texts. Oxford.

Fedotoff, A.

1996 Motif of Miraculous Birth in Mongolian and Korean Myths and Epic, International Journal of Central Asian Studies 1: 201-223.
FRANKFORT, H.

2001 Reyes y dioses. Madrid.

GASTER, T. H.

1987 Heroes, en Eliade, M. (ed.): The Encyclopedia of Religions, Vol. VI, New York: 302-305.

JunG, C. G.; KeRÉNYI K.

2004 Introducción a la esencia de la mitología. Madrid.

Hornung, E.

1999 El uno y los múltiples. Concepciones egipcias de la divinidad. Barcelona.

LESKO, L. H.

1972 The ancient Egyptian Book of Two Ways, Berkeley, Los Angeles, London.

\section{LicthTHeim, M.}

1997 Moral Values in Ancient Egypt. Fribourg.

LÓPEZ, J.

2005 Cuentos y fábulas del antiguo Egipto. Barcelona.

Moret, A.

1931 La légende d'Osiris à l'époque thébaine d'après l'hymne à Osiris du Louvre, BIFAO 30: 725-750.

Naville, E.

1886 Das Ägyptische Todtenbuch der XVIII. bis XX. Dynastie. Berlin.

PARKInSON, R.

1991 The Tale of the Elocuent Peaseant. Oxford.

Sethe, K.

1959 Ägyptische Lesestücke zum Gebrauch in akademischen Unterricht. Leipzig. 


\section{Trabajos de Egiptología Papers on Ancient Egypt}

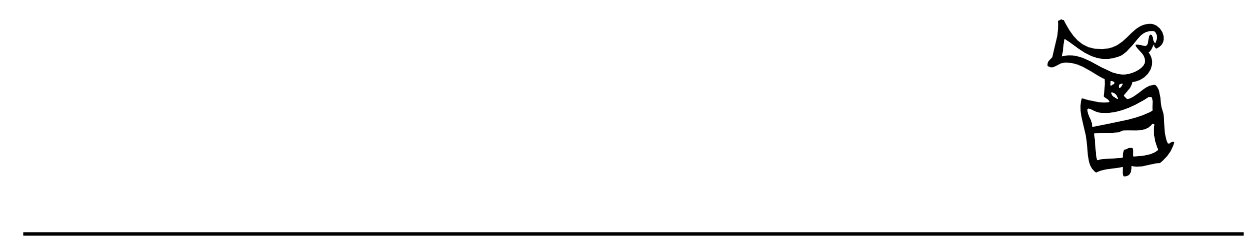

Número 5/1 2009 


\section{Actas \\ III Congreso Ibérico de Egiptología III Congresso Ibérico de Egiptologia}

Editores

Miguel Ángel Molinero Polo Covadonga Sevilla Cueva 


\title{
Editor
}

Miguel Ángel Molinero Polo

Universidad de La Laguna

\section{Consejo Editorial}

\author{
Antonio Pérez Largacha \\ Universidad de Castilla-La Mancha \\ José-R. Pérez-Accino \\ Birkbeck, Universidad de Londres \\ Covadonga Sevilla Cueva \\ Universidad Autónoma de Madrid
}

\section{Comité Científico}

Josep Cervelló i Autuori

Universitat Autònoma de Barcelona

$\mathrm{M}^{\mathrm{a}}$ José lópez Grande

Universidad Autónoma de Madrid

Josep Padró i Parcerisa

Universitat de Barcelona

$\mathrm{M}^{\mathrm{a}}$ Carmen Pérez Die

Museo Arqueológico Nacional, Madrid

Ester Pons Mellado

Museo Arqueológico Nacional, Madrid

José M. Serrano Delgado

Universidad de Sevilla

\section{Colaboradores Editoriales}

Linda Steynor

English editorial assistant

Hervé Mouriacoux

Assistant éditorial pour la langue française 
Trabajos de Egiptología está producida por Isfet. Egiptología e Historia c/ Blanco $1,2^{\circ}$

38400 Puerto de la Cruz

Tenerife-Islas Canarias

España

Maquetación: Proyecto Limón

(C) Autores de los artículos aparecidos

y Consejo Editorial de Trabajos de Egiptología - Papers on ancient Egypt

Depósito Legal: TF-2302-2009

ISSN: $1695-4750$

Imprime: Gráfica Los Majuelos, S.L.L.

imprenta@graficaslosmajuelos.com

Tfno.: 922311455 


\section{Comité Científico \\ III Congreso Ibérico de Egiptología III Congresso Ibérico de Egiptologia}

Miguel Á. Molinero Polo

Universidad de La Laguna

Presidente del Comité Organizador del III Congreso Ibérico de Egiptología

Miembro del Comité Organizador del I Encuentro de Egiptología

Josep Cervelló Autuori

Universitat Autònoma de Barcelona

Presidente del Comité Organizador del II Congreso Ibérico de Egiptologia

José Manuel Galán Allué

Consejo Superior de Investigaciones Cientificas

Director del Proyecto Djehuty, Luxor, Egipto

$\mathrm{M}^{\mathrm{a}}$ Helena Trindade Lopes

Universidad de Lisboa

Directora de la Misión Arqueológica Portuguesa en Menfis

Josep Padró i Parcerisa

Universitat de Barcelona

Director de la Misión Arqueológica de Oxirrinco

Antonio Pérez Largacha

Universidad de Castilla - La Mancha

Miembro del Comité Organizador del I Encuentro de Egiptología

José Ramón Pérez-Accino

Birkbeck College, University of London

Miembro del Comité Organizador del I Encuentro de Egiptología

$\mathrm{M}^{\mathrm{a}}$. Carmen Pérez Díe

Museo Arqueológico Nacional

Directora de la Misión Arqueológica Española en Heracleópolis Magna, Egipto

Covadonga Sevilla Cueva

Universidad Autónoma de Madrid

Miembro del Comité Organizador del I Encuentro de Egiptología 